\title{
A Phase I, Randomized, Double-Blind, Single-Dose, Placebo-Controlled Safety, Tolerability, And Pharmacokinetic/Pharmacodynamic Study Of Evolocumab In Healthy Chinese Subjects
}

This article was published in the following Dove Press journal:

Clinical Pharmacology: Advances and Applications

Chao Liu ID
Hong Lu'
Fei Yuan'
Wei-Li Chen'
Hong-Rong Xu'
Hui Li
Cheng-Pang Hsu ${ }^{3}$
Ogo Egbuna
Jihua Wu ${ }^{2}$
Clapton Dias ${ }^{3}$
Bassam Abosaleem ${ }^{3}$
Jitesh Rana ${ }^{3}$
Maria Laura Monsalvo ${ }^{3}$
Xue-Ning Li ${ }^{1}$
Zhigang Yu ${ }^{4}$
'Department of Clinical Pharmacology,
Zhongshan Hospital, Fudan University,
Shanghai, People's Republic of China;
${ }^{2}$ Clinical Pharmacology and Early
Development, Amgen China R \& D Center,
Shanghai, People's Republic of China;
${ }^{3}$ Research \& Development, Amgen Inc.,
Thousand Oaks, CA, USA; ${ }^{4}$ Clinical
Development, Amgen China R \& D Center,
Shanghai, People's Republic of China

Correspondence: Xue-Ning Li Department of Clinical Pharmacology, Zhongshan Hospital, Fudan University, I 80 Fenglin Road, Shanghai 200032

People's Republic of China

Tel +8621 60267666

Email li.xuening@zs-hospital.sh.cn

Zhigang Yu

Research \& Development, Amgen Inc.,

Thousand Oaks, CA 91320, USA

Tel +l 805 447-9384

Email zhigangy@amgen.com
Purpose: Evolocumab is a human monoclonal antibody that reduces circulating low-density lipoprotein cholesterol (LDL-C) by inhibiting proprotein convertase subtilisin/kexin type 9 (PCSK9). Data on evolocumab pharmacokinetics and pharmacodynamics are derived mostly from Caucasian populations. The objectives of this study were to characterize the single-dose pharmacokinetic and pharmacodynamic parameters, safety, and tolerability of evolocumab in healthy Chinese subjects.

Subjects and methods: This was a phase 1, randomized, double-blind, placebo-controlled study (CTR20150465). Two parallel cohorts were randomized 5:1 to receive single subcutaneous injections of either evolocumab $(140 \mathrm{mg}$ or $420 \mathrm{mg}$ ) or placebo. Pharmacokinetics, pharmacodynamics, and safety were evaluated through day 85 . The primary endpoints were maximum concentration $\left(\mathrm{C}_{\max }\right)$ and area under the drug concentration-time curve from time 0 to time of last quantifiable concentration $\left(\mathrm{AUC}_{\text {last }}\right)$.

Results: Thirty-six men (median age 26) were enrolled to receive evolocumab $140 \mathrm{mg}$ $(n=15)$, evolocumab $420 \mathrm{mg}(\mathrm{n}=15)$, or placebo $(\mathrm{n}=6)$. After $140 \mathrm{mg}$ and $420 \mathrm{mg}$ evolocumab, mean (SD) $\mathrm{C}_{\max }$ was $13.8(3.6 \mu \mathrm{g} / \mathrm{mL}$ and $67.6(15.2) \mu \mathrm{g} / \mathrm{mL}$, respectively, and mean (SD) $\mathrm{AUC}_{\text {last }}$ was $166(55)$ day $\mu \mathrm{g} / \mathrm{mL}$ and $1110(274)$ day $\mu \mathrm{g} / \mathrm{mL}$, respectively. LDL-C declined reversibly, with reductions of $70 \%$ at $140 \mathrm{mg}$ and $71 \%$ at $420 \mathrm{mg}$. Maximum effects on LDL-C and PCSK9 levels were reached by day 15 and $24 \mathrm{hrs}$, respectively, at $140 \mathrm{mg}$, and by day 22 and $4 \mathrm{hrs}$, respectively, at $420 \mathrm{mg}$. No serious adverse events occurred and the overall incidence of treatment-emergent adverse events was similar for evolocumab and placebo: $26.7 \%$ (140 mg) and 33.3\% (placebo); 66.7\% (420 mg) and 66.7\% (placebo).

Conclusion: In this population of healthy Chinese subjects, single $140 \mathrm{mg}$ and $420 \mathrm{mg}$ doses of evolocumab exhibited nonlinear kinetics and more than dose-proportional increases in exposure, were associated with up to $71 \%$ reduction in LDL-C, and demonstrated a safety profile similar to placebo.

Keywords: cardiovascular disease, homozygous familial hypercholesterolemia, PCSK9 inhibitors, monoclonal antibodies, ethnic sensitivity

\section{Introduction}

Cardiovascular disease (CVD) is the primary cause of death in both the developed and developing worlds, accounting for approximately $30 \%$ of all deaths and $46 \%$ of the deaths from noncommunicable diseases worldwide. ${ }^{1,2}$ In China, CVD is the cause of over $40 \%$ of all deaths. ${ }^{3}$ A large proportion of CVD is due to 
atherosclerosis. Dyslipidemia is a major, modifiable risk factor for atherosclerosis and CVD, including coronary heart disease. In patients with a high risk of CVD, Chinese guidelines for the management of dyslipidemia recommend moderate-intensity statins to lower low-density lipoprotein cholesterol (LDL-C) and reduce cardiovascular events. ${ }^{4}$ As some patients cannot achieve adequate lipid control with the use of statins or are unable to tolerate any statin or an effective dose of statins, alternative treatment options are needed. ${ }^{5}$

Statin therapy is modestly effective in reducing LDL-C concentrations in patients with homozygous hypercholesterolemia $(\mathrm{HoFH}){ }^{6-8}$ Mutations in plasma proprotein convertase subtilisin/kexin type 9 (PCSK9) were discovered in a French family with FH in 2003. ${ }^{9}$ Patients with FH have higher levels of PCSK9 compared with non-FH controls, and statin treatment causes an increase in PCSK9 in these patients, particularly those with HoFH. ${ }^{10}$ Statin-induced increases in PCSK9, therefore, blunt the extent of LDL lowering because PCSK9 binding to the LDL-receptor (LDL-R) causes the complex to undergo lysosomal degradation, resulting in less LDL-R on the cell surface.

Evolocumab is a human monoclonal immunoglobulin G2 that specifically binds to PCSK9. ${ }^{11}$ This interaction prevents PCSK9 from binding to the LDL-R, which results in increased LDL-R expression and a subsequent decrease in circulating concentrations of LDL-C. Evolocumab has demonstrated LDL-C reduction of approximately $60 \%$ across a variety of patient populations on stable lipid-lowering therapy in global clinical trials including those with $\mathrm{FH} .{ }^{12}$ In China, evolocumab was approved in July 2018 as an adjunct to diet and other LDL-lowering therapies (eg, statins, ezetimibe, LDL apheresis) for the treatment of patients with HoFH who require additional lowering of LDL-C and in January 2019 to reduce the risk of cardiovascular events (myocardial infarction, stroke, and coronary revascularization) in adults with established atherosclerotic CVD. ${ }^{13}$ In the United States, evolocumab is also indicated for the treatment of primary hyperlipidemia and mixed dyslipidemia to further reduce LDL-C as an adjunct to diet alone or in combination with a maximally tolerated statin and/or with other lipid-lowering therapies. ${ }^{13}$

The majority of pharmacokinetic and pharmacodynamic data on evolocumab derive from mostly Caucasian populations. ${ }^{14-17}$ The objectives of the present study were to characterize the single-dose pharmacokinetic and pharmacodynamic parameters, safety, and tolerability of evolocumab administered subcutaneously in healthy Chinese subjects.

\section{Materials And Methods}

\section{Study Design}

This was a phase 1, single-dose, randomized, doubleblind, placebo-controlled study (study 20120134; CTR20150465). Baseline LDL-C and PCSK9 were determined at screening. Two parallel cohorts of subjects (18 subjects per cohort) were enrolled and randomized in a 5:1 ratio to receive either evolocumab or placebo. Cohort 1 received a single subcutaneous injection of $140 \mathrm{mg}$ evolocumab or placebo using an autoinjector/pen, while cohort 2 received subcutaneous injection of $420 \mathrm{mg}$ evolocumab or placebo using three autoinjector/pens. Randomization was based on a randomization schedule provided by an independent randomization group at Amgen before the start of the study. This study was conducted in accordance with the International Council for Harmonisation Good Clinical Practice, China Good Clinical Practice, and Human Genetic Resource Administration of China regulations/guidelines. The protocol was reviewed by the institutional review board at Zhongshan Hospital. All subjects provided written informed consent before undergoing any study-related procedures, including screening procedures.

\section{Subjects}

Eligible subjects were healthy men and women residing in China and of Chinese ancestry, $\geq 18$ and $\leq 45$ years of age, with a body mass index $\geq 18$ and $\leq 32 \mathrm{~kg} / \mathrm{m}^{2}$ and a calculated LDL-C concentration $\geq 70 \mathrm{mg} / \mathrm{dL}$ and $\leq 190 \mathrm{mg} / \mathrm{dL}$ at the time of screening. Women were required to be of nonreproductive potential and were not to be pregnant or lactating. Subjects agreed to maintain their current general diet and physical activity regimens. Exclusion criteria included creatinine kinase $>3$ times the upper limit of normal (ULN) at screening or day -1 , long QT interval, smoking more than a half pack per day, unwillingness to refrain from strenuous exercise for $72 \mathrm{hrs}$ prior to study visits, unwillingness to limit alcohol consumption during the study, and use of medications or supplements (to be reviewed by investigator).

\section{Assays}

After overnight fasting, blood samples were collected into tubes and centrifuged after clotting; serum aliquots were stored at $-80^{\circ} \mathrm{C}$. Unbound serum evolocumab was 
measured using a validated enzyme-linked immunosorbent assay (ELISA) with limits of quantification between 0.8 and $10 \mu \mathrm{g} / \mathrm{mL} .^{17}$ Unbound serum PCSK9 concentrations were measured by an ELISA assay. ${ }^{18}$ At screening, calculated LDL-C concentration was analyzed by a local laboratory. At all other time points, LDL-C concentration was measured at a central laboratory using preparative ultracentrifugation. LDL-C values were converted to SI units by multiplying $\mathrm{mg} / \mathrm{dL}$ by 0.02586 . Testing for binding anti-evolocumab antibodies was performed by ELISA assay before dosing and at day 85; if a subject tested positive for binding antibodies, they were evaluated for neutralizing antibodies.

\section{Statistical Considerations}

The primary endpoints were pharmacokinetic parameters of evolocumab (maximum concentration $\left[\mathrm{C}_{\max }\right]$ and area under the drug concentration-time curve from time 0 to time of last quantifiable concentration [ $\left.\mathrm{AUC}_{\text {last }}\right]$ ). For the $140 \mathrm{mg}$ cohort, assuming a coefficient of variation $(\mathrm{CV})$ of $80.7 \%$ for $\mathrm{C}_{\max }$ and $136.4 \%$ for $\mathrm{AUC}_{\text {last }}$ from a study evaluating evolocumab inter-patient pharmacokinetic variability, expected precision of the estimated geometric means was 1.38 and 1.59 , respectively. For the $420 \mathrm{mg}$ cohort, assuming a CV of $60.0 \%$ for $\mathrm{C}_{\max }$ and $69.9 \%$ for $\mathrm{AUC}_{\text {last, }},{ }^{15,19}$ expected precision of the estimated geometric means was 1.29 and 1.33 , respectively.

Secondary endpoints included treatment-emergent adverse events (AEs), vital signs, electrocardiograms (ECGs), laboratory safety tests, pharmacodynamic parameters, area under the effect curve for LDL-C from day 1 to

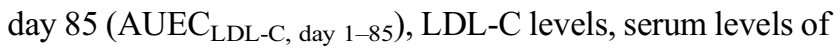
PCSK9, and additional pharmacokinetic parameters including, but not limited to, time of maximum concentration $\left(\mathrm{t}_{\mathrm{max}}\right)$.

The pharmacokinetic analysis set included all subjects for whom at least one evolocumab pharmacokinetic parameter or endpoint could be adequately estimated; the safety analysis set included all subjects who received at least one dose of investigational product (evolocumab or placebo). Subjects were summarized according to the actual treatment received and analyses were descriptive. Pharmacokinetic analyses were performed using Phoenix WinNonlin version 6.4.

AEs were coded using the Medical Dictionary for Regulatory Activities, version 19.1. AEs were graded for severity using the Common Terminology Criteria for Adverse Events (CTCAE) version 4.0. AEs associated with injectable protein therapies (hypersensitivity reactions and injection-site reactions) were prespecified events of interest; AEs associated with drug-induced hepatic disorders were also evaluated. The AEs of interest were analyzed by narrow and broad searches using either standardized or Amgen-defined search strategies of Medical Dictionary for Regulatory Activities queries.

\section{Results}

\section{Subjects}

The first subject was enrolled in October 2016 and the last subject completed the study in March 2017. Thirty-six subjects were enrolled and randomized, received their assigned dose, and completed the study. The safety analysis set included 36 subjects. All 36 subjects were men and the median (range) age was $26(22,36)$ years (Table 1). At baseline, the mean (SD) LDL-C concentration was 2.66 (0.47) $\mathrm{mmol} / \mathrm{L}$ and the mean (SD) PCSK9 concentration was $222(53) \mathrm{ng} / \mathrm{mL}$.

\section{Pharmacokinetic Parameters}

The pharmacokinetic analysis set consisted of 509 evolocumab serum samples from the 30 subjects who received evolocumab (15 in each cohort). All 30 subjects were included in the noncompartmental analysis, and no samples

Table I Demographic And Baseline Characteristics

\begin{tabular}{|c|c|c|c|c|c|}
\hline & \multicolumn{2}{|l|}{ Cohort I } & \multicolumn{2}{|l|}{ Cohort 2} & \multirow{2}{*}{$\begin{array}{l}\text { All } \\
(n=36)\end{array}$} \\
\hline & $\begin{array}{l}\text { Placebo } \\
(n=3)\end{array}$ & $\begin{array}{l}\text { I40 mg evolocumab } \\
(n=\mid 5)\end{array}$ & $\begin{array}{l}\text { Placebo } \\
(n=3)\end{array}$ & $\begin{array}{l}420 \mathrm{mg} \text { evolocumab } \\
(n=15)\end{array}$ & \\
\hline Male, n (\%) & $3(100)$ & $15(100)$ & $3(100)$ & $15(100)$ & $36(100)$ \\
\hline Age, median years (range) & $27(24,29)$ & $27(22,36)$ & $26(25,29)$ & $25(22,33)$ & $26(22,36)$ \\
\hline BMI $\left(\mathrm{kg} / \mathrm{m}^{2}\right)$, mean $(\mathrm{SD})$ & $23.9(1.5)$ & $22.8(2.3)$ & $27.6(2.2)$ & $22.7(2.6)$ & $23.3(2.7)$ \\
\hline LDL-C (mmol/L) $)^{\mathrm{a}}$, mean (SD) & $2.66(0.47)$ & $2.74(0.4 \mathrm{I})$ & $2.46(0.4 \mathrm{I})$ & $2.6(0.54)$ & $2.66(0.47)$ \\
\hline PCSK9, ng/mL, mean (SD) & $225(56)$ & $244(55)$ & $212(38)$ & 201 (49) & $222(53)$ \\
\hline
\end{tabular}

Note: ${ }^{\text {a }} \mathrm{SI}$ units were calculated by multiplying $\mathrm{mg} / \mathrm{dL}$ by 0.02586 .

Abbreviations: BMI, body mass index; LDL-C, low-density lipoprotein cholesterol; PCSK9, proprotein convertase subtilisin/kexin type 9. 
were excluded from the noncompartmental analysis or summary statistics. Serum evolocumab concentration-time profiles are shown in Figure 1 and serum evolocumab pharmacokinetic parameters are shown in Table 2.

After administration of a single subcutaneous $140 \mathrm{mg}$ dose of evolocumab, the mean (SD) $\mathrm{C}_{\max }$ and $\mathrm{AUC}_{\text {last }}$ were 13.8 (3.6) $\mu \mathrm{g} / \mathrm{mL}$ and 166 (55) day $\mu \mathrm{g} / \mathrm{mL}$, respectively. The median time to reach $\mathrm{C}_{\max }\left(\mathrm{t}_{\max }\right)$ was 4.0 days. The mean (SD) values for area under the drug concentration-time curve from time 0 to infinity $\left(\mathrm{AUC}_{\text {inf }}\right)$ and halflife were 183 (41) day $\mu \mathrm{g} / \mathrm{mL}$ and 4.6 (1.2) days, respectively, at $140 \mathrm{mg}$. After administration of a single subcutaneous $420 \mathrm{mg}$ dose of evolocumab, the mean (SD) exposures based on $\mathrm{C}_{\max }$ and $\mathrm{AUC}_{\text {last }}$ were $67.6(15.2) \mu \mathrm{g} /$ $\mathrm{mL}$ and 1110 (274) day $\mu \mathrm{g} / \mathrm{mL}$, respectively, and the median $\mathrm{t}_{\max }$ was 6.0 days. The mean (SD) values for $\mathrm{AUC}_{\mathrm{inf}}$ and half-life were 1130 (276) day $\mu \mathrm{g} / \mathrm{mL}$ and 10.1 (2.0) days, respectively, at $420 \mathrm{mg}$.

\section{Pharmacodynamic Parameters}

Pharmacodynamic parameters were measured in the safety analysis set. The geometric mean percentage change in

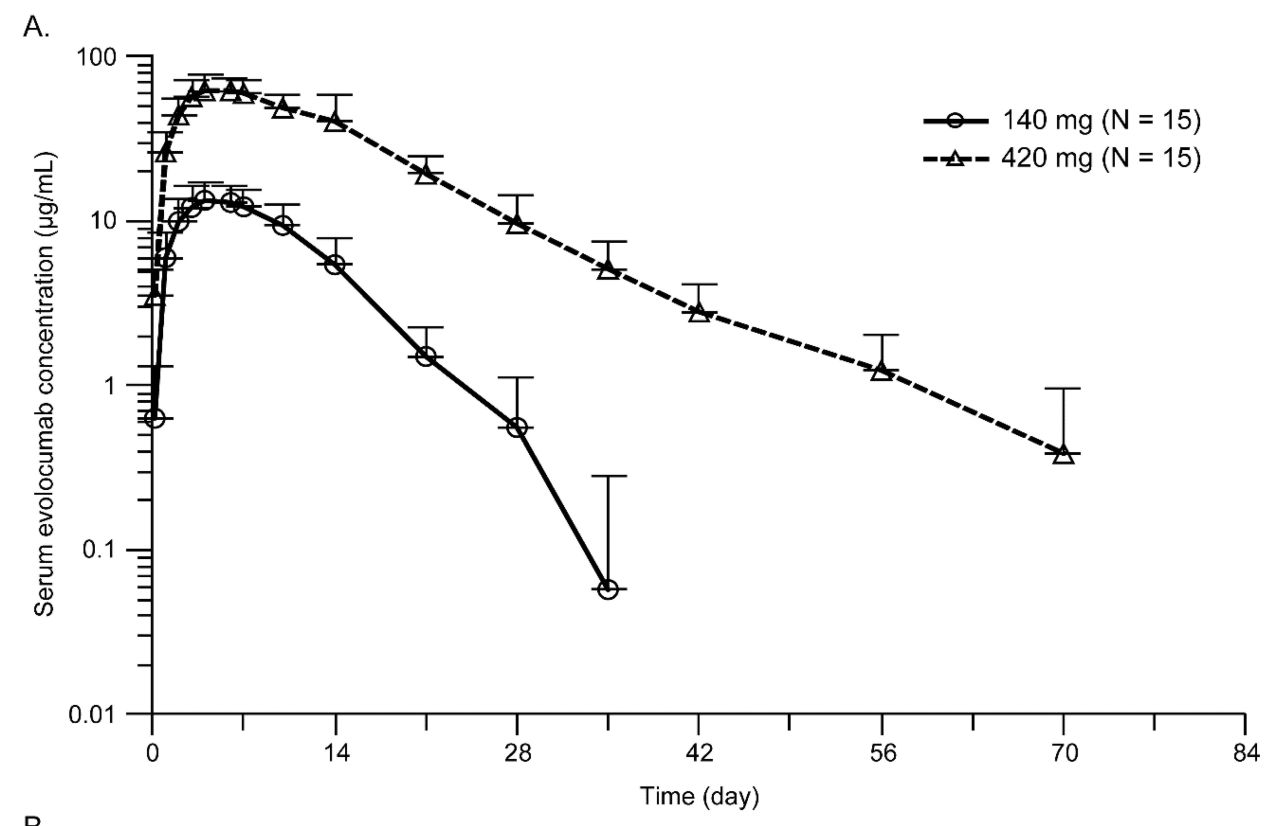

B.

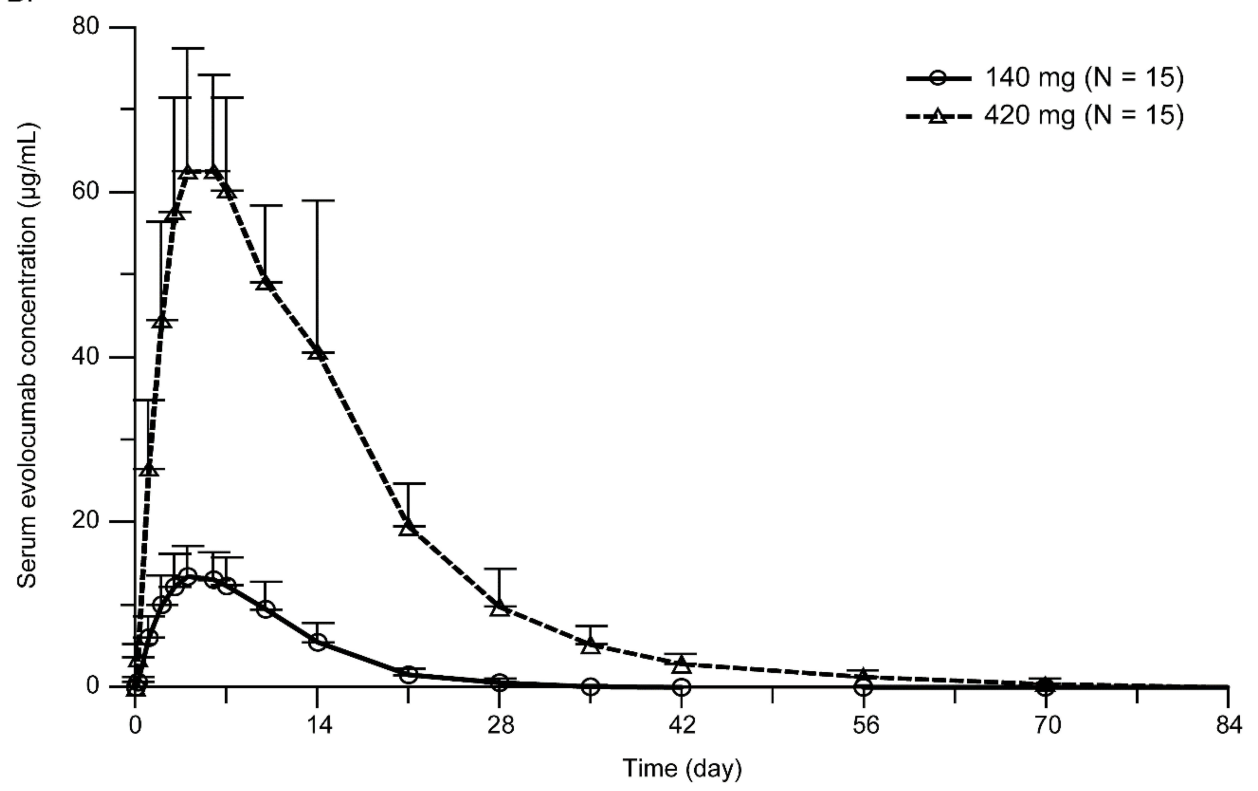

Figure I Mean (SD) serum evolocumab concentration-time profiles after single-dose subcutaneous administration. (A) Linear-log. (B) Linear-linear. 
Table 2 Estimated Pharmacokinetic Parameters After Single-Dose Subcutaneous Administration Of Evolocumab

\begin{tabular}{|c|c|c|}
\hline & 140 mg Evolocumab $(n=\mid 5)$ & $420 \mathrm{mg}$ Evolocumab $(n=15)$ \\
\hline$t_{\max }$, median (range) days & $4(3,6)$ & $6(3,14)$ \\
\hline $\mathrm{C}_{\max }$, mean $(\mathrm{SD}) \mu \mathrm{g} / \mathrm{mL}$ & I3.8 (3.6) & $67.6(15.2)$ \\
\hline $\mathrm{AUC}_{\text {last }}$, mean $(\mathrm{SD})$ day $\cdot \mu \mathrm{g} / \mathrm{mL}$ & $166(55)$ & $1110(274)$ \\
\hline $\mathrm{AUC}_{\text {inf, }}$ mean (SD) day $\cdot \mu \mathrm{g} / \mathrm{mL}$ & $183(4 I)^{\mathrm{a}}$ & $1130(276)$ \\
\hline$t_{1 / 2, z}$, mean $(S D)$ days & $4.6(1.2)^{\mathrm{a}}$ & $10.1(2.0)$ \\
\hline
\end{tabular}

Note: ${ }^{a}$ Based on $\mathrm{n}$ of 14 subjects; the terminal phase in one subject could not be defined.

Abbreviations: AUC, area under the drug concentration-time curve; $A \cup C_{\text {inf, }} A U C$ from time 0 to infinity; $A \cup C_{\text {last }}$, $A U C$ from time 0 to time of last quantifiable concentration; $C_{\max }$, maximum observed drug concentration; $t_{1 / 2, z}$, terminal half-life; $t_{\max }$, time to reach $C_{\max }$.

ultracentrifugation LDL-C is shown in Figure 2A. In both and maximum LDL-C reduction was reached by day 15 in evolocumab groups, reductions in LDL-C levels were the evolocumab $140 \mathrm{mg}$ group and day 22 in the $420 \mathrm{mg}$ observed by the first post-baseline assessment on day 2 group. In cohort 1, the mean percentage change in LDL-C
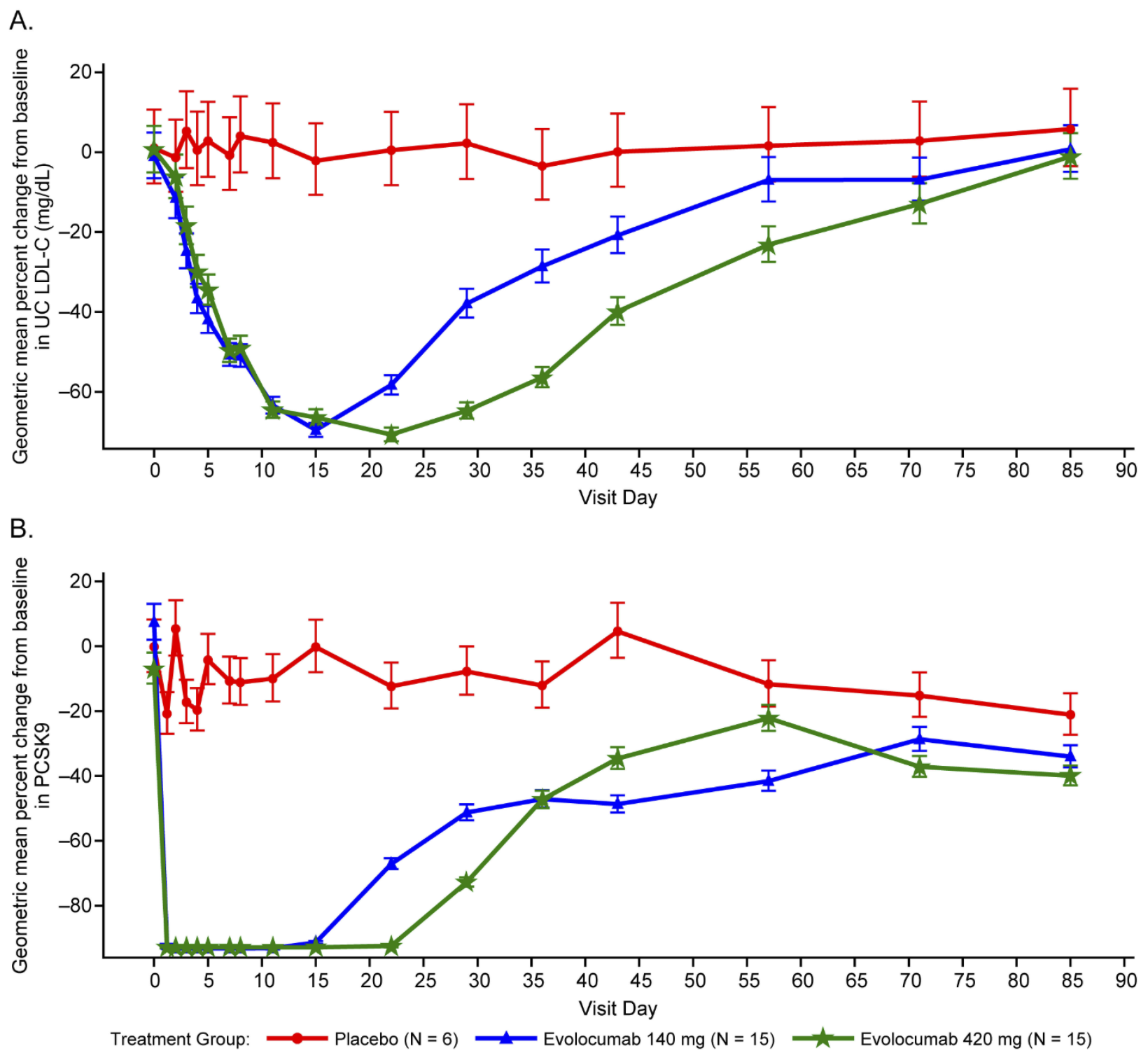

Figure 2 Geometric mean (SE) percentage change from baseline in pharmacodynamic parameters. (A) Ultracentrifugation low-density lipoprotein cholesterol (LDL-C). (B) Proprotein convertase subtilisin/kexin type 9 (PCSK9). 
from baseline to day 15 was $-69.6 \%$ in the $140 \mathrm{mg}$ evolocumab group and $-1.7 \%$ in the placebo group. In cohort 2 , the mean percentage change from baseline to day 22 was $-70.7 \%$ in the $420 \mathrm{mg}$ evolocumab group and $3.7 \%$ in the placebo group. By day 29 , the mean percentage change from baseline was $-37.9 \%$ in the $140 \mathrm{mg}$ evolocumab group and $-64.7 \%$ in the $420 \mathrm{mg}$ evolocumab group. At the end of the study, mean LDL-C levels were near baseline levels in all groups $(0.8 \%$ change for $140 \mathrm{mg}$ evolocumab, $3.1 \%$ for cohort 1 placebo, $-1.1 \%$ for $420 \mathrm{mg}$ evolocumab, and $8.5 \%$ for cohort 2 placebo). The

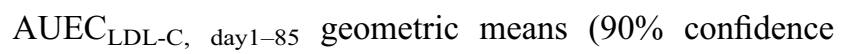
interval [CI]) for the $140 \mathrm{mg}$ and $420 \mathrm{mg}$ evolocumab groups were $170.97(156.27,187.06)$ day $\cdot \mathrm{mmol} / \mathrm{L}$ and $134.90(120.74,150.71)$ day $\cdot \mathrm{mmol} / \mathrm{L}$, respectively.

The geometric mean percentage change in PCSK9 is shown in Figure 2B. In both evolocumab groups, PCSK9 levels decreased substantially within $4 \mathrm{hrs}$ postdose on day 1 (the first post-baseline assessment), with mean percentage changes from baseline to $4 \mathrm{hrs}$ postdose of $-92.2 \%$ and $-92.9 \%$ in the $140 \mathrm{mg}$ and $420 \mathrm{mg}$ evolocumab groups, respectively, and $-24.2 \%$ and $-17.4 \%$ in cohort 1 and 2 placebo groups, respectively. PCSK9 levels reached the nadir, which was near the lower limit of quantification (15 ng/mL), by $24 \mathrm{hrs}$ for $140 \mathrm{mg}$ and by $4 \mathrm{hrs}$ for $420 \mathrm{mg}$ evolocumab. PCSK9 levels then remained around the nadir through day 15 for $140 \mathrm{mg}$ evolocumab and through day 22 for $420 \mathrm{mg}$ evolocumab. By day 22, the mean percentage change from baseline was $-67.2 \%$ for $140 \mathrm{mg}$ evolocumab; by day 29, the mean percentage change from baseline was $-72.7 \%$ for $420 \mathrm{mg}$ evolocumab. At the end of the study, mean percentage changes from baseline were $-34.1 \%$ and $-39.9 \%$ for $140 \mathrm{mg}$ and $420 \mathrm{mg}$ evolocumab, respectively.

\section{Safety}

Treatment-emergent AEs were reported for four subjects $(26.7 \%)$ in the $140 \mathrm{mg}$ evolocumab group, one subject $(33.3 \%)$ in the cohort 1 placebo group, 10 subjects $(66.7 \%)$ in the $420 \mathrm{mg}$ evolocumab group, and two subjects $(66.7 \%)$ in the cohort 2 placebo group (Table 3$)$. No serious or fatal AEs were reported during the study. All AEs were CTCAE grade 1 or 2, with the exception of one grade 3 treatment-related AE of serum creatine phosphokinase $>5 \times \mathrm{ULN}$, which occurred on day 57 without symptoms and resolved within 14 days without intervention (140 mg evolocumab). Treatment-related AEs occurred in three subjects $(20.0 \%)$ in the $140 \mathrm{mg}$ evolocumab group, one subject $(33.3 \%)$ in the cohort 1 placebo group, three subjects $(20.0 \%)$ in the $420 \mathrm{mg}$ evolocumab group, and two subjects $(66.7 \%)$ in the cohort 2 placebo group. AEs of interest included potential hypersensitivity

Table 3 Treatment-Emergent Adverse Events

\begin{tabular}{|c|c|c|c|c|}
\hline \multirow[t]{2}{*}{ n (\%) } & \multicolumn{2}{|l|}{ Cohort I } & \multicolumn{2}{|l|}{ Cohort 2} \\
\hline & $\begin{array}{l}\text { Placebo } \\
(n=3)\end{array}$ & $\begin{array}{l}\text { I } 40 \mathrm{mg} \text { Evolocumab } \\
(\mathrm{n}=15)\end{array}$ & $\begin{array}{l}\text { Placebo } \\
(n=3)\end{array}$ & $\begin{array}{l}420 \mathrm{mg} \text { Evolocumab } \\
(\mathrm{n}=15)\end{array}$ \\
\hline $\begin{array}{l}\text { Number of subjects reporting treatment-emergent } \\
\text { adverse events }\end{array}$ & I (33.3) & $4(26.7)$ & $2(66.7)$ & $10(66.7)$ \\
\hline Blood creatine phosphokinase increased & I (33.3) & $2(13.3)$ & I (33.3) & I (6.7) \\
\hline Urine protein present & $0(0.0)$ & $2(13.3)$ & $0(0.0)$ & $0(0.0)$ \\
\hline Nasopharyngitis & $0(0.0)$ & I (6.7) & $0(0.0)$ & $\mathrm{I}(6.7)$ \\
\hline Blood potassium increased & $0(0.0)$ & I (6.7) & $0(0.0)$ & $0(0.0)$ \\
\hline Gastric disorder & $0(0.0)$ & I (6.7) & $0(0.0)$ & $0(0.0)$ \\
\hline Urticaria & $0(0.0)$ & I (6.7) & $0(0.0)$ & $0(0.0)$ \\
\hline Varicella & $0(0.0)$ & I (6.7) & $0(0.0)$ & $0(0.0)$ \\
\hline Upper respiratory tract infection & $0(0.0)$ & $0(0.0)$ & $0(0.0)$ & $6(40.0)$ \\
\hline Alanine aminotransferase increased & $0(0.0)$ & $0(0.0)$ & $0(0.0)$ & $2(13.3)$ \\
\hline Aspartate aminotransferase increased & $0(0.0)$ & $0(0.0)$ & $0(0.0)$ & $2(13.3)$ \\
\hline White blood cell count increased & $0(0.0)$ & $0(0.0)$ & $0(0.0)$ & $2(13.3)$ \\
\hline Blood bilirubin elevated & $0(0.0)$ & $0(0.0)$ & I (33.3) & I (6.7) \\
\hline Chalazion & $0(0.0)$ & $0(0.0)$ & $0(0.0)$ & I (6.7) \\
\hline Glomerular filtration rate decreased & $0(0.0)$ & $0(0.0)$ & $0(0.0)$ & I (6.7) \\
\hline Intervertebral disc protrusion & $0(0.0)$ & $0(0.0)$ & $0(0.0)$ & I (6.7) \\
\hline Tinea pedis & $0(0.0)$ & $0(0.0)$ & $0(0.0)$ & I (6.7) \\
\hline
\end{tabular}


reactions, injection-site reactions, and drug-induced hepatic disorder events. One potential hypersensitivity event of grade 1 urticaria occurred on day 37 and resolved within 3 days following application of topical ointment (140 mg evolocumab). Four subjects had potential drug-induced hepatic disorder AEs, including two subjects with elevated alanine aminotransferase (ALT) and aspartate aminotransferase (AST), and two subjects with elevated blood bilirubin. Both subjects with ALT/AST increases had received $420 \mathrm{mg}$ evolocumab: one subject had an ALT laboratory value $>3 \times \mathrm{ULN}$ (but $<5 \times \mathrm{ULN}$ ) and $\mathrm{AST}<3 \times \mathrm{ULN}$ on day 85 and was treated with hepatoprotective medication with the events resolving after 65 days; the other subject had both AST and ALT laboratory values $<3 \times$ ULN on day 29 , which resolved after 14 days without intervention. In the two subjects with elevated blood bilirubin (both $<2 \times$ ULN occurring in isolation without transaminase increases), one subject (420 mg evolocumab) had events on days 2 and 29, and the other (placebo) had an event on day 85 ; the events resolved without intervention in both subjects. No potential injection-site reactions were reported. No clinically important effects of evolocumab were observed on selected laboratory parameters, ECGs, or vital signs. Among the 30 subjects who received evolocumab, there were no binding or neutralizing antibodies at any time during the study.

\section{Discussion}

In this double-blind, placebo-controlled study of single doses of evolocumab in healthy Chinese subjects, single doses of $140 \mathrm{mg}$ and $420 \mathrm{mg}$ were associated with nonlinear kinetics and more than dose-proportional increases in exposure, along with rapid, reversible reductions in PCSK 9 followed by LDL-C of $70 \%$ and $71 \%$, respectively. No notable differences in the safety profiles were observed across the placebo and evolocumab groups in this population of Chinese subjects. The results of this study are similar to those reported in mostly Caucasian subjects, ${ }^{15-17}$ suggesting that evolocumab is not associated with differing sensitivity across Caucasian and Chinese populations.

Like most monoclonal antibodies, evolocumab is eliminated through both nonspecific (immunoglobulin $\mathrm{G}$ catabolism) and specific target-mediated mechanisms (binding to PCSK9). ${ }^{14,15}$ The pharmacokinetics of evolocumab showed nonlinear elimination in the dose range studied (140 mg and $420 \mathrm{mg}$ ) in Chinese subjects, consistent with the model of target-mediated drug disposition for evolocumab previously demonstrated in modeling based on mostly Caucasian subjects. ${ }^{14}$

After $140 \mathrm{mg}$ evolocumab administration, mean (SD) exposures based on $\mathrm{C}_{\max }$ and $\mathrm{AUC}_{\text {last }}$ in Chinese subjects were 13.8 (3.6) $\mu \mathrm{g} / \mathrm{mL}$ and 166 (55) day· $\mu \mathrm{g} / \mathrm{mL}$, respectively, comparable to those reported in mostly Caucasian subjects of $18.6(7.3) \mu \mathrm{g} / \mathrm{mL}$ and 188 (98.6) day $\mu \mathrm{g} / \mathrm{mL}$, respectively. ${ }^{16}$ After $420 \mathrm{mg}$ evolocumab administration, mean (SD) exposures based on $\mathrm{C}_{\max }$ and $\mathrm{AUC}_{\text {last }}$ in Chinese subjects were 67.6 (15.2) $\mu \mathrm{g} / \mathrm{mL}$ and 1110 (274) day $\cdot \mu \mathrm{g} / \mathrm{mL}$, respectively, also comparable to those reported in mostly Caucasian subjects of 59.0 (17.2) $\mu \mathrm{g} /$ $\mathrm{mL}$ and 924 (346) day $\mu \mathrm{g} / \mathrm{mL}$, respectively. ${ }^{16}$ Thus, evolocumab exposure was more than dose-proportional and similar across ethnicities, confirming the conclusions of population models. ${ }^{17,20}$

Peak reductions in LDL-C were reached after 15 and 22 days with the $140 \mathrm{mg}$ and $420 \mathrm{mg}$ evolocumab doses, respectively, in Chinese subjects, comparable to 14 and 21 days, respectively, reported in mostly Caucasian subjects. ${ }^{16}$ Maximal reductions in LDL-C were up to $71 \%$ in Chinese subjects compared with $66 \%$ in mostly Caucasian subjects. ${ }^{14,17}$ Thus, as observed in Caucasian subjects, the $140 \mathrm{mg}$ and $420 \mathrm{mg}$ doses produced a similar therapeutic effect on LDL-C lowering in Chinese subjects.

The safety profile of $140 \mathrm{mg}$ and $420 \mathrm{mg}$ evolocumab observed in this study was similar to that of the placebo control groups, with no notable differences between groups in the type or severity of AEs observed. Most AEs were isolated incidences and were mild or moderate in severity and there were no serious events and no binding or neutralizing anti-evolocumab antibodies. These findings are similar to those reported in mostly Caucasian subjects, in which there were no notable differences in safety profiles between the placebo and evolocumab groups. ${ }^{13,15}$ Therefore, based on the present sample, the safety profile of evolocumab in Chinese subjects appears to be consistent with that reported in Caucasian subjects.

Body weight and target concentration are established variables affecting elimination of monoclonal antibodies. For most monoclonal antibodies, ethnic differences across Japanese and non-Japanese populations have not been of sufficient magnitude to warrant different doses. ${ }^{21,22}$ The lack of differences in the pharmacokinetics and pharmacodynamics of evolocumab across Asian and Caucasian populations was confirmed by population modeling analyses ${ }^{20}$ in 
which data were pooled from five studies (the present study, one additional study in Chinese subjects [ $\mathrm{Lu}$ et al, manuscript in preparation, Supplemental File], one in Japanese subjects, ${ }^{20}$ and two in Caucasian subjects ${ }^{16}$ ). This model showed that evolocumab pharmacokinetic/pharmacodynamic parameters, including the absorption rate constant $\left(\mathrm{K}_{\mathrm{a}}\right)$, clearance, volume, and concentration associated with half-maximal inhibition $\left(\mathrm{IC}_{50}\right)$, were not significantly different between the Caucasian and Asian populations. ${ }^{20}$ Similarly, a large population pharmacokinetic/pharmacodynamic model including 5474 mostly Caucasian subjects from 11 evolocumab clinical studies showed that differences in subject factors such as body weight, PCSK9 concentration, and ethnicity were not sufficient to warrant dose adjustment. ${ }^{17}$

Single doses of $140 \mathrm{mg}$ and $420 \mathrm{mg}$ showed similar efficacy for LDL-C reduction in the present study. Consistent with this finding, in global phase 3 studies, evolocumab $140 \mathrm{mg}$ every 2 weeks (Q2W) and $420 \mathrm{mg}$ monthly (QM) dosing had clinically equivalent effects over time on LDL-C. ${ }^{23}$ Evolocumab $140 \mathrm{mg}$ Q2W and evolocumab $420 \mathrm{mg}$ QM are approved and commercially available doses for the treatment of adults with primary hypercholesterolemia or mixed dyslipidemia and adults with established atherosclerotic cardiovascular disease.

Evolocumab is often used as an additional therapy in patients who are receiving maximally tolerated statin therapy and require further LDL-C lowering or who have had cardiovascular events despite achieving desired LDL-C levels on statins. Statins act by inhibiting 3-hydroxy-3-methylglutaryl coenzyme A reductase, which reduces the production of cholesterol and lowers intracellular cholesterol levels. ${ }^{24}$ PCSK9 antibody inhibitors including evolocumab inhibit circulating PCSK9, thereby preventing LDL-C receptor degradation. Both statins, by increasing LDL-receptor expression, and evolocumab, by permitting LDL-receptors to recycle back to the liver cell surface, increase the number of LDL receptors available to clear LDL from the bloodstream.

Considering that some patients continue to experience cardiovascular risk while receiving statin therapy and given that non-statin treatment options have modest efficacy and/or are poorly tolerated, there is an unmet medical need for a potent, effective non-statin treatment that will further reduce cardiovascular risk through LDL-C reduction. Combining novel therapies such as PCSK9 inhibitors with statins provides new options for further LDL-C lowering.

\section{Conclusion}

In conclusion, in this population of healthy Chinese subjects, single $140 \mathrm{mg}$ and $420 \mathrm{mg}$ doses of evolocumab exhibited nonlinear kinetics and more than dose-proportional increases in exposure, were associated with rapid and reversible reductions in LDL-C and PCSK9, and demonstrated a safety profile similar to placebo. These findings were consistent with evolocumab pharmacokinetics, pharmacodynamics, safety, and tolerability reported previously in mostly Caucasian subjects.

\section{Data Sharing Statement}

Qualified researchers may request data from Amgen clinical studies. Complete details are available at the following: http://www.amgen.com/datasharing.

\section{Acknowledgments}

This study was sponsored by Amgen Inc. Wanda J Krall, $\mathrm{PhD}$, provided editorial support funded by Amgen. Chao Liu and Hong $\mathrm{Lu}$ are co-first authors for this study. Dr Zhigang Yu's current affiliation is Amgen Inc, Thousand Oaks, CA, USA.

\section{Disclosure}

Xue-Ning Li, Fei Yuan, Wei-Li Chen, Hong-Rong Xu, Hui $\mathrm{Li}$, Chao Liu are employees of the Department of Clinical Pharmacology, Zhongshan Hospital, Fudan University. Hong Lu, Cheng-Pang Hsu, Bassam Abosaleem, Jitesh Rana, Maria Laura Monsalvo, and Zhigang Yu are employees of Amgen and hold Amgen securities. Ogo Egbuna, Jihua $\mathrm{Wu}$, and Clapton Dias were employees of Amgen while the study was conducted. The authors report no other conflicts of interest in this work.

\section{References}

1. Roth GA, Johnson C, Abajobir A, et al. Global, regional, and national burden of cardiovascular diseases for 10 causes, 1990 to 2015. J Am Coll Cardiol. 2017;70(1):1-25. doi:10.1016/j.jacc.2017.04.052

2. Organization WH. Global status report on noncommunicable diseases 2014; 2014. Available from: https://www.who.int/nmh/publications/ncdstatus-report-2014/en/. Accessed February 19, 2019.

3. Chen WW, Gao RL, Liu LS, et al. China cardiovascular diseases report 2015: a summary. J Geriatr Cardiol. 2017;14(1):1-10. doi:10.11909/j. issn.1671-5411.2017.01.012

4. Revision Jcfg. 2016 Chinese guidelines for the management of dyslipidemia in adults. J Geriatr Cardiol. 2018;15(1):1-29. doi:10.11909/j. issn.1671-5411.2018.01.011

5. Krähenbühl S, Pavik-Mezzour I, von Eckardstein A. Unmet needs in LDL-C lowering: when statins won't do! Drugs. 2016;76(12):11751190. doi:10.1007/s40265-016-0613-0

6. Marais AD, Raal FJ, Stein EA, et al. A dose-titration and comparative study of rosuvastatin and atorvastatin in patients with homozygous familial hypercholesterolaemia. Atherosclerosis. 2008;197(1):400 406. doi:10.1016/j.atherosclerosis.2007.06.028 
7. Raal FJ, Pilcher GJ, Illingworth DR, et al. Expanded-dose simvastatin is effective in homozygous familial hypercholesterolaemia. Atherosclerosis. 1997;135(2):249-256. doi:10.1016/s0021-9150(97) 00168-8

8. Gagné C, Gaudet D, Bruckert E, Group ES. Efficacy and safety of ezetimibe coadministered with atorvastatin or simvastatin in patients with homozygous familial hypercholesterolemia. Circulation. 2002;105(21):2469-2475. doi:10.1161/01.cir.0000018744.58460.62

9. Abifadel M, Varret M, Rabès JP, et al. Mutations in PCSK9 cause autosomal dominant hypercholesterolemia. Nat Genet. 2003;34 (2):154-156. doi:10.1038/ng1161

10. Raal F, Panz V, Immelman A, Pilcher G. Elevated PCSK9 levels in untreated patients with heterozygous or homozygous familial hypercholesterolemia and the response to high-dose statin therapy. $J$ Am Heart Assoc. 2013;2(2):e000028. doi:10.1161/JAHA.112.000028

11. Langslet G, Emery M, Wasserman SM. Evolocumab (AMG 145) for primary hypercholesterolemia. Expert Rev Cardiovasc Ther. 2015;13 (5):477-488. doi:10.1586/14779072.2015.1030395

12. Wiggins BS, Senfield J, Kassahun H, Lira A, Somaratne R. Evolocumab: considerations for the management of hyperlipidemia. Curr Atheroscler Rep. 2018;20(4):17. doi:10.1007/s11883-018-0720-3

13. REPATHA ${ }^{\circledR}$ (evolocumab) [prescribing Information]. Shanghai: Amgen China; 2018

14. Gibbs JP, Doshi S, Kuchimanchi M, et al. Impact of target-mediated elimination on the dose and regimen of evolocumab, a human monoclonal antibody against proprotein convertase subtilisin/kexin type 9 (PCSK9). J Clin Pharmacol. 2017;57(5):616-626. doi:10.1002/ jcph. 840

15. Dias CS, Shaywitz AJ, Wasserman SM, et al. Effects of AMG 145 on low-density lipoprotein cholesterol levels: results from 2 randomized, double-blind, placebo-controlled, ascending-dose phase 1 studies in healthy volunteers and hypercholesterolemic subjects on statins. $J \mathrm{Am}$ Coll Cardiol. 2012;60(19):1888-1898. doi:10.1016/j.jacc.2012.08.986

16. Kasichayanula S, Grover A, Emery MG, et al. Clinical pharmacokinetics and pharmacodynamics of evolocumab, a PCSK9 inhibitor. Clin Pharmacokinet. 2018;57(7):769-779. doi:10.1007/s40262-0170620-7
17. Kuchimanchi M, Grover A, Emery MG, et al. Population pharmacokinetics and exposure-response modeling and simulation for evolocumab in healthy volunteers and patients with hypercholesterolemia. J Pharmacokinet Pharmacodyn. 2018;45(3):505-522. doi:10.1007/ s10928-018-9592-y

18. Colbert A, Umble-Romero A, Prokop S, Xu R, Gibbs JP, Pederson S. Characterization of a quantitative method to measure free proprotein convertase subtilisin/kexin type 9 in human serum. MAbs. 2014;6 (4):1103-1113. doi:10.4161/mabs.28719

19. Teramoto T, Shaywitz A, Hamilton L. A phase 1, randomized, double-blind, placebo-controlled study of AMG 145 comparing the pharmacokinetics, pharmacodynamics, and safety in healthy Japanese and Caucasian subjects. Paper presented at: proceedings of 45 th annual scientific meeting of the Japan Atherosclerosis Society 2013; Tokyo.

20. Wang C, Zheng Q, Zhang M, Lu H. Lack of ethnic differences in the pharmacokinetics and pharmacodynamics of evolocumab between Caucasian and Asian populations. Br J Clin Pharmacol. 2019;85 (1):114-125. doi:10.1111/bcp.13767

21. Matsushima S, Huang Y, Suzuki H, Nishino J, Lloyd P. Ethnic sensitivity assessment - pharmacokinetic comparability between Japanese and non-Japanese healthy subjects on selected mAbs. Expert Opin Drug Metab Toxicol. 2015;11(2):179-191. doi:10.15 17/17425255.2015.990438

22. Chiba K, Yoshitsugu H, Kyosaka Y, et al. A comprehensive review of the pharmacokinetics of approved therapeutic monoclonal antibodies in Japan: are Japanese phase I studies still needed? J Clin Pharmacol. 2014;54(5):483-494. doi:10.1002/jcph.231

23. Stroes E, Robinson JG, Raal FJ, et al. Consistent LDL-C response with evolocumab among patient subgroups in PROFICIO: a pooled analysis of 3146 patients from phase 3 studies. Clin Cardiol. 2018;41 (10):1328-1335. doi:10.1002/clc.23049

24. Stancu C, Sima A. Statins: mechanism of action and effects. J Cell Mol Med. 2001;5(4):378-387. doi:10.1111/j.1582-4934.2001.tb0 0172.x
Clinical Pharmacology: Advances and Applications is an international, peer-reviewed, open access journal publishing original research, reports, reviews and commentaries on all areas of drug experience in humans. The manuscript management system is completely online and includes a very quick and fair peer-review system, which is all easy to use. Visit http://www.dovepress.com/testimonials.php to read real quotes from published authors.

Submit your manuscript here: https://www.dovepress.com/clinical-pharmacology-advances-and-applications-journal 